\title{
Normalisierung oder Mobilisierung? - Die Auswirkungen politischer Online-Kommunikation auf die Wahlbeteiligung am Beispiel einer Internet-Applikation zur Bundestagswahl 2009
}

\author{
Stefan Marschall / Martin Schultze
}

\begin{abstract}
Normalization or Mobilization? - The Effects of Political Online Communication on Voter Turnout: The Case of an Internet-Application at the 2009 German Federal Election Abstract: Regarding the effects of political internet communication on political participation, two positions oppose each other: While the normalization thesis contends that online communication reinforces already existing disparities within political participation, the mobilization thesis assumes that online communication can mobilize individuals who are not interested in politics. The paper contributes empirically to this debate by analyzing the effect of a popular online application, "Wahl-O-Mat", on voting intention at the 2009 German Federal Election. The findings support the mobilization thesis.
\end{abstract}

Keywords: Voting Intention, Internet/Online Communication, Mobilization, "Voting Advice Applications”, "German Longitudinal Election Study”

Schlüsselwörter: Wahlbeteiligung, Internet-/Online-Kommunikation, Mobilisierung, „Voting Advice Applications“, „German Longitudinal Election Study“

\section{Einleitung ${ }^{1}$}

In den Wahlkämpfen der vergangenen Jahre ist politische Internet-Kommunikation zu einem obligatorischen Baustein und das ,Netz der Netze ${ }^{6}$ zu einem Ort politischen Marketings geworden (Chadwick u. Howard 2009; Schweitzer u. Albrecht 2011). Der Online-Kommunikation werden beachtliche Mobilisierungspotenziale zugesprochen, die zu einer Zunahme der Partizipation im politischen System und dabei auch zur Steigerung der Beteiligung an Wahlen führen könnten (Pautz 2010; Zittel u. Fuchs 2007). So sind neben die Netzaktivitäten parteipolitischer Akteure zunehmend Initiativen überparteilicher Akteure getreten, die auf die Erhöhung der Wahlbeteiligung zielen. Innerhalb der Gruppe von Internet-Angeboten, die von non-party actors in Wahlkämpfen bereitgestellt werden (Farrell u. Schmitt-Beck 2008), haben Voting Advice Applications (VAAs) erhebliche Verbreitung und Nachfrage erfahren. Bei VAAs handelt es sich um Web-Tools, die die Policy-Positionen der Nutzerinnen und Nutzer ${ }^{2}$ mit denen der zur Wahl antretenden Parteien oder Kandidaten vergleichen (vgl. als Überblick Cedroni u. Garzia 2010). Nachdem Wähler ihre Positionen zu einer Reihe von Thesen markiert ha-

1 Für hilfreiche Anmerkungen danken wir insbesondere den anonymen PVS-Gutachter/-innen sowie der PVS-Redaktion.

2 Aus Gründen der Lesbarkeit werden im Folgenden nur noch die maskulinen Formen verwendet. 
ben, gleichen VAAs das Antwortmuster der Nutzer mit denen der antretenden Parteien/Kandidaten ab und zeigen auf dieser Grundlage an, welche Partei oder welcher Kandidat dem jeweiligen Wähler am nächsten steht.

Die deutsche VAA-Variante, der Wahl-O-Mat, der von der Bundeszentrale für politische Bildung produziert wird, ist seit der Bundestagswahl 2002 im Einsatz. In Folge wurde er bei den Bundestagswahlen 2005 und 2009, bei den Europawahlen 2004 und 2009 sowie bei zahlreichen Landtagswahlen verwendet. Bereits seine erste Version zur Bundestagswahl 2002 erfuhr eine starke Nachfrage (ca. 3,6 Millionen Nutzungen), welche sich seitdem nahezu stetig erhöht hat; bei der letzten Wahl zum Deutschen Bundestag 2009 wurde er 6,7 Millionen Mal durchgespielt (Marschall 2011, S. 41). Es ist davon auszugehen, dass ein beachtlicher Teil des deutschen Elektorats dieses Angebot bereits einmal genutzt hat.

Auch in anderen Ländern haben sich in den vergangenen Jahren Voting Advice Applications etabliert (Cedroni 2010). Eines der ältesten und erfolgreichsten Tools findet sich mit dem Stemwijzer in den Niederlanden. Dort nutzten bei der Tweede-Kamer-Wahl von 200640 Prozent der Wähler die Voting Advice Application (De Graaf 2010, S. 26). Anlässlich der Europawahl 2009 trug überdies das Projekt „EU-Profiler“ des Europäischen Hochschulinstituts Florenz, das für alle 27 Staaten der Europäischen Union sowie für Kroatien, die Türkei und die Schweiz eine VAA-Version produziert hatte, zur weiteren Verbreitung des Tools bei (Ladner et al. 2010; Trechsel u. Mair 2009).

Aufgrund ihres Erfolges haben VAAs mittlerweile die Aufmerksamkeit sozialwissenschaftlicher Forschung gefunden: Voting Advice Applications sind in den vergangenen Jahren immer wieder zum Gegenstand wissenschaftlicher Untersuchungen, Tagungen und Publikationen geworden (zusammenfassend Cedroni u. Garzia 2010). Trotz diverser Forschungsinitiativen handelt es sich bei VAAs in der Gesamtschau jedoch noch um ein untererforschtes Phänomen, vor allem was ihre Wirkungen auf das politische Verhalten betrifft.

Angesichts der intensiven Nachfrage nach Voting Advice Applications in zahlreichen Staaten stellt sich die Frage, inwiefern die Nutzung von VAAs Einfluss auf ihre Nutzer nimmt - insbesondere hinsichtlich ihrer Beteiligung an Wahlen (Garzia 2010). Verändert das Nutzen von VAAs die Bereitschaft, zur Wahl zu gehen, und wenn ja, in welche Richtung und unter welchen Bedingungen? Antworten auf diese Fragen erlauben wiederum vorbehaltliche Rückschlüsse auf das Mobilisierungspotenzial von Online-Kommunikation generell.

Die Frage nach der Wirkung von VAAs auf die Wahlbeteiligung soll für den Wahl-O-Mat auf der Grundlage von Daten der „German Longitudinal Election Study“ (GLES)3 zur Bundestagswahl 2009 beantwortet werden. Die GLES-Daten erlauben einen systematischen Vergleich zwischen Onlinern, die den Wahl-O-Mat genutzt haben, und den Nicht-Nutzern innerhalb der Gruppe der Onliner. Damit ermöglicht der zur Verfügung stehende Datensatz zu überprüfen, ob ein genuiner VAA-Effekt existiert, der unabhängig von der soziodemografischen Zusammensetzung, den spezifischen politischen Einstellungen der Nutzer sowie den sonsti-

3 Ein Überblick über Ziele und Struktur der GLES findet sich bei Schmitt-Beck et al. 2010. 
gen Kommunikationseinflüssen auf die Nutzerschaft dieser Tools ist, und inwiefern dieser Effekt auf die Wahlbeteiligungsabsicht wirkt.

Im folgenden Abschnitt wird zunächst der Diskussionsstand zu Wirkungen von Online-Kommunikation auf die politische Partizipation im Spannungsfeld zwischen der Normalisierungs- und der Mobilisierungsthese herausgearbeitet und die davon abgeleitete Relevanz von VAAs und möglicher VAA-Effekte thematisiert. Bereits vorliegende Studien, die erste Aussagen über die Wirkung der Nutzung von VAAs auf die Wahlbeteiligung treffen, werden dargestellt und diskutiert. Anschließend präsentieren wir Ergebnisse einer multivariaten Analyse der GLESDaten zur Wirkung des Wahl-O-Mat bei der Bundestagswahl 2009. Auf der Grundlage der Datenanalyse können wir die Existenz eines VAA-Effekts - kontrolliert für relevante Drittvariablen - überprüfen und damit Schlussfolgerungen über die Wirkung der Nutzung von Voting Advice Applications auf die Wahlbeteiligung ziehen. Abschließend werden die Befunde mit Blick auf die Kontroverse zwischen Normalisierungs- und Mobilisierungsthese eingeordnet und auf verbleibende Forschungsdesiderate verwiesen.

\section{Theoretischer Rahmen und Forschungsstand: Normalisierung oder Mobilisierung durch Internet-Kommunikation und VAAs}

Das wachsende Online-Engagement parteipolitischer wie auch parteineutraler Akteure vor Wahlen beruht auf der Unterstellung, dass sich politische InternetKommunikation auf Quantität und Qualität des Wahlverhaltens auswirkt - auch dann, wenn der Anteil der politischen Kommunikation im Internet im Verhältnis zur Netzaktivität der Bürger insgesamt einen geringen Umfang einnimmt (Emmer 2005; Emmer u. Wolling 2010).

Untersuchungen zu den Effekten politischer Online-Kommunikation kommen zu unterschiedlichen Schlüssen, ob ein solcher Effekt existiert, die in zwei konträre Thesen münden. Gemäß der „Normalisierungsthese“ nutzen vor allem Bürger, die ohnehin politisch aktiv sind, auch das Internet für politische Kommunikation (vgl. Chadwick 2006; Zittel u. Freund 2011), womit neue Informationstechnologien lediglich den Status quo der Offline-Partizipationsungleichheit fortschreiben. Das Internet hat demnach keinen originären Effekt auf die Wahlbeteiligung oder Wahlentscheidung. Beispielsweise stellen Drew und Weaver (2006) in einer Studie für die US-Präsidentschaftswahlen 2004 fest, dass - kontrolliert für demografische Merkmale und traditionelle Medieneinwirkung - die Rezeption von InternetNachrichten keinen signifikanten Einfluss auf die Wahlbeteiligungsabsicht hat. Johnson und Kaye (2003) kommen zu dem Schluss, dass politische Internetnutzung weder Effekte auf die Wahlbeteiligung noch auf die konkrete Wahlentscheidung bei der US-Präsidentschaftswahl 2000 ausübte. Schmitt-Beck und Mackenrodt (2009) halten für die deutschen Bundestagswahlen 2005 sogar einen negativen Einfluss fest: Zwischen zwei Panelwellen sank bei Nutzern, die intensiv politische Inhalte im Internet konsumiert hatten, die Wahlbeteiligungsabsicht.

Demgegenüber besagt die „Mobilisierungsthese“, dass ansonsten schwer erreichbare, kaum politisch aktive Gruppen durch das Internet mobilisiert werden 
(Gibson et al. 2005). So zeigen Tolbert und McNeal (2003), dass der Mobilisierungseffekt des Internets selbst unter Kontrolle des sozioökonomischen Status und der klassischen Mediennutzung bestehen bleibt und sich signifikant positiv auf die Wahlteilnahme auswirkt. Emmer und Vowe kommen zu dem Schluss, dass zwar bei einer Reihe von politischen Aktivitäten das Vorhandensein eines Internet-Zugangs keine Rolle spielt, aber ein messbarer Anteil häufiger in Form interpersonaler Kommunikation politisch partizipiert, sobald er über einen InternetZugang verfügt (2004, S. 206).

Bei unterschiedlicher Spezifikation gehen beide, die Normalisierungs- wie die Mobilisierungsthese, davon aus, dass Online-Kommunikation auf das politische Verhalten der Nutzer wirkt, es also einen „Medieneffekt“ (Lin 2009) von Internet-Anwendungen gibt. Ob und wie Internet-Kommunikation auf das Wahlverhalten wirkt, wird in der empirischen Forschung freilich für verschiedene Nutzergruppen unterschiedlich beantwortet (vgl. Abold 2006; Emmer et al. 2006). Das Wirkungsverhältnis kann alternativ und ergänzend entlang einzelner InternetAnwendungen und ihrer spezifischen Effekte differenziert untersucht werden (vgl. z. B. Hirzalla et al. 2011; Pautz 2010).

Eine solche applikationsscharfe Wirkungsanalyse ist für die folgende Ermittlung eines VAA-Effektes leitend. Die Wirkung von VAAs auf die Wahlbeteiligung soll in einem ersten Schritt unter Berücksichtigung der speziellen Eigenschaften des Tools plausibel gemacht werden, bevor in einem zweiten Schritt auf bereits vorliegende empirische Ergebnisse rekurriert wird.

Dass VAAs überhaupt Einfluss auf die Wahlbeteiligung nehmen, wird seitens der Betreiber dieser Internet-Angebote ausdrücklich angestrebt. Voting Advice Applications werden in erster Linie als Instrumente der politischen Bildungsarbeit angeboten, die Bürger zur Auseinandersetzung mit und Teilnahme an der Wahl mobilisieren sollen. ${ }^{4}$

Die Existenz solcher Nutzungseffekte von Voting Advice Applications kann unter Rückgriff auf Rational-Choice-Ansätze (Downs 1957; Caballero 2005) in Verbindung mit dem Konzept der Issue-Orientierung plausibilisiert werden, die in der empirischen Wahlforschung als kurzfristiger Erklärungsfaktor für die Wahlbeteiligung und Wahlentscheidung eine wichtige Rolle spielt (Fuchs u. Rohrschneider 2005; Kunz u. Thaidigsmann 2005; Kellermann u. Rattinger 2007). ${ }^{5}$ Gerade bei der Bundestagswahl 2009 haben - angesichts der Wirtschafts- und Finanzkrise sowie kaum unterscheidbarer Spitzenkandidaten - Issue-Orientierungen einen beachtlichen Einfluss auf die Wahlentscheidung ausgeübt (Rudi 2011, S. 189). Eine Voraussetzung für die Orientierungs- und Mobilisierungskraft von Sachfragen ist es, dass Themen wahrgenommen, als wichtig eingeschätzt und mit Parteien und ihren Positionen in Verbindung gebracht werden (vgl. Kunz u. Thaidigsmann 2005). Der hierzu erforderliche Prozess der Informationsbeschaffung und -aus-

4 Dieses Ziel findet sich bei der Bundeszentrale für politische Bildung, die den Wahl-O-Mat bereitstellt, unter: http://www.bpb.de/methodik/KZ6IKY,0,Fakten_zum_WahlOMat.html (Stand: 18.03.2012).

5 Mit Verwendung des Konzepts „Issue-Orientierung“ (synonym: Sachfragenorientierung) greifen wir auch auf Elemente des sozialpsychologischen Ansatzes der Wahlforschung zurück (Campbell et. al 1960, Lewis-Beck et al. 2008; Schoen und Weins 2005). 
wertung ist für den individuellen Wähler mit erheblichem Aufwand, insbesondere mit Zeitkosten, verbunden (Johann 2009). VAAs bieten vor anstehenden Wahlen eine effiziente Form der Orientierung entlang politischer Sachfragen und verbinden diese mit den Parteien, indem sie eine Anzahl von wahlkampfrelevanten Policy-Fragen auswählen und auf Thesenform reduzieren sowie zugleich die entsprechenden Positionen der zur Wahl antretenden Parteien erfassen und auswerten. ${ }^{6}$ In ihrer Ergebnisdarstellung ermitteln und illustrieren VAAs die issue-basierte Nähe zwischen den Wählern und den Positionen der Parteien. Aufgrund ihrer Funktionalität können VAAs die Informationsbeschaffungs- und Informationsverarbeitungskosten für ihre Nutzer senken und als Konsequenz einer optimierten Kosten-Nutzen-Relation die Wahlteilnahme wahrscheinlicher machen (Garzia 2010, S. 19).

Indem sie die Positionen der Parteien erfassen und mit denen der Nutzer abgleichen, arbeiten VAAs zugleich die Policy-Differenzen zwischen den Parteien heraus und machen diese für die Wähler unterscheidbarer. So berichten 64,4 Prozent der befragten Nutzer unmittelbar nach dem Spielen des Tools, dass ihnen die Unterschiede zwischen den beteiligten Parteien deutlicher geworden sind (Marschall 2011, S. 44). Dies kann sich positiv auf die Wahlbeteiligung auswirken, da der Grad an wahrgenommener Unterscheidbarkeit zwischen den zur Wahl antretenden Parteien einen positiven Einfluss auf die Wahlbereitschaft ausübt (Faas 2010).

Kurzum: Aus einer theoretischen Perspektive kann ein positiver VAA-Effekt auf die Wahlbeteiligung zweifach plausibilisiert werden: (1) mit Verweis auf die Senkung von Informationsbeschaffungs- und Verarbeitungskosten durch das Tool, (2) mit Bezug auf die VAA-generierte höhere Unterscheidbarkeit der Parteien für die Wähler.

Dass VAAs tatsächlich diese plausible Wirkung entfalten, wird von einigen vorliegenden Studien empirisch untermauert. Dabei unterscheiden sich die Analysen darin, ob sie die Mobilisierungswahrscheinlichkeit messen, den Anteil der Mobilisierten taxieren, den Mobilisierungseffekt auf das Gesamtelektorat beziehen und/ oder die Normalisierungs- respektive Mobilisierungsthese für verschiedene Subgruppen untersuchen. So haben Mykkänen und Moring (2006) ermittelt, dass die Nutzung einer VAA bei den finnischen Parlamentswahlen 2003 die Wahrscheinlichkeit, dass die jeweiligen Nutzer zur Wahl gehen, um 20 Prozent erhöht hat. Marschall und Schmidt machen diese mobilisierende Wirkung bei dem Einsatz der deutschen VAA vor den Europawahlen 2009 hingegen nur bei rund 10 Prozent der Nutzer aus (2010). Ladner und Pianzola (2010, S. 219-220) schätzen, dass bei den Wahlen in der Schweiz 2007 circa 15 Prozent der Nutzer der Schweizer VAA „smartvote“ durch die Nutzung angeregt wurden, zur Wahl zu gehen, und dass smartvote bezogen auf das Gesamtelektorat für rund 1 Prozent aller Stimmabgaben verantwortlich war. Boogers (2006) taxiert die Anzahl derer, die

6 Dass sowohl der Auswahl- als auch der redaktionelle Verarbeitungsprozess zugleich mit Informationsverlusten und -verzerrungen verbunden ist, soll hier nicht weiter verfolgt werden (s. z. B. zur Thesenselektion die Kritik bei Walgrave et al. 2009, vgl. zur weiteren Kritik an der Methode des Tools Ramonaite 2010). 
aufgrund der Nutzung des niederländischen Stemwijzers 2006 zur Wahl motiviert wurden, auf 11 Prozent. Hirzalla et al. (2011) finden - ebenfalls mit Daten der niederländischen Wahlstudie zur Parlamentswahl 2006 - sowohl empirische Unterstützung für die Normalisierungsthese mit Fokus auf Personengruppen über 25 Jahre als auch Mobilisierungseffekte bei Personen, die 25 Jahre und jünger sind.

Diese erheblichen Unterschiede in den Befunden zum Einfluss von VAAs auf die Wahlbeteiligung bzw. Wahlbeteiligungsabsicht können in der Varianz der politischen Rahmenbedingungen der jeweiligen Länder begründet sein. So ist denkbar, dass die mobilisierende Wirkung der VAAs vom jeweiligen Wahl- und Parteiensystem abhängig ist: In Mehrheitswahlsystemen mit nur wenigen Parteien, die eine realistische Einzugschance in das Parlament haben, müsste der Orientierungsbedarf und infolge das Mobilisierungspotenzial von VAAs geringer ausfallen als in politischen Systemen mit einer Vielzahl solcher Parteien (vgl. dazu auch Wall et al. 2009, S. 205).

Die heterogenen Befunde können aber auch damit zusammenhängen, dass bei den empirischen Erhebungen kein einheitliches methodisches Vorgehen gewählt worden ist, respektive dieses Vorgehen mitunter auf instabilen Füßen steht. Die Daten, auf denen Forschungsergebnisse zu VAAs und ihrer Wirkung auf die Wahlbeteiligung basieren, sind bei einer Reihe von Untersuchungen direkt nach dem Spielen des Tools erhoben worden („Exit-Befragungen“); entweder sind die Nutzer aktiv zur Teilnahme an einer Befragung eingeladen worden (Pop-up-/LayerFenster); oder auf der Ergebnisseite konnte eine Schaltfläche angeklickt werden, die zur Befragung führte. Beide Vorgehensweisen sind als Rekrutierungsverfahren für Online-Umfragen mit einer Reihe von Einschränkungen bezüglich der Repräsentativität des Samples verbunden (siehe beispielsweise Maurer u. Jandura 2009; Schnell et al. 2008, S. 377-386; Taddicken 2007; Zerback et al. 2009) - es sei denn, diese wird nach bestimmten Kriterien überprüft oder wiederhergestellt.

Eine weitere Fehlerquelle könnte sein, dass in zahlreichen Studien subjektiv vermutete Wirkungen des Tools erfasst werden, d. h. spekulative und prädiktive Aussagen über zukünftiges Verhalten herangezogen werden, die unter dem unmittelbaren Eindruck des Spielens einer VAA stehen. Walgrave et al. (2008) haben in einer Studie kontrolliert, inwieweit die Selbstauskünfte unmittelbar nach der Nutzung einer VAA mit dem tatsächlichen späteren Wahlverhalten in Übereinstimmung stehen. Sie kamen zu dem Ergebnis, dass nur ein Drittel derjenigen, die in Exit-Befragungen angaben, sie würden aufgrund der VAA-Empfehlung ihre Wahlabsicht ändern, dies tatsächlich auch taten (vgl. auch Kleinnijenhuis u. van Hoof 2008). Schließlich kann bei Exit-Surveys keine Vergleichsgruppe von Nicht-VAASpielern generiert werden, die bei der Ermittlung von originären VAA-Effekten hilfreich sein mag (vgl. z. B. Nuytemans et al. 2010). ${ }^{7}$ Insofern spricht vieles dafür, die Validität der vorliegenden Befunde zur Wirkung von VAAs auf die Wahlbeteiligung mit einem alternativen methodischen Ansatz zu überprüfen.

Zusammenfassend lässt sich konstatieren, dass trotz der dargestellten methodischen Einschränkungen zahlreiche und deutliche Hinweise auf einen positiven

7 Auch in Studien, deren Datengrundlage die Möglichkeit des Vergleichs zwischen Nutzern und Nicht-Nutzern bieten würde, ist dies nicht praktiziert worden (z. B. Mykkänen u. Moring 2006). 
Einfluss von VAAs auf die Wahlbeteiligung bzw. Wahlbeteiligungsabsicht vorliegen. Dies gilt es im Folgenden zu überprüfen: In unserer Modellierung, die sich an vorherrschende Wirkungstheorien und -studien zu diesen Applikationen anschließt, ist die Nutzung der VAA die unabhängige und die Wahlbeteiligungsabsicht die abhängige Variable (Garzia 2010). Mit Blick auf die vorliegenden empirischen Befunde und theoretischen Annahmen gehen wir folglich davon aus, dass sich mit den GLES-Daten zur Bundestagswahl 2009 ein positiver Effekt der WahlO-Mat-Nutzung (unabhängige Variable) auf die Wahlbeteiligungsabsicht (abhängige Variable) nachweisen lässt.

\section{Empirische Analyse}

\subsection{Datengrundlage und Bildung von Vergleichsgruppen}

Für die Beantwortung der Frage nach einem VAA-Effekt wenden wir uns den Nutzern des Wahl-O-Mat anlässlich der Bundestagswahl 2009 zu und greifen auf eine Vorwahlumfrage der "German Longitudinal Election Study“ mit dem Schwerpunkt Wahlkampf ${ }^{8}$ zurück, die Fragen zur Nutzung der deutschen VAA beinhaltet. Bei dem herangezogenen GLES-Modul handelt es sich um eine OnlineUmfrage mit standardisiertem Fragebogen, die unmittelbar vor der Bundestagswahl 2009 durchgeführt worden ist (18. bis 26. September 2009). Grundgesamtheit der Befragten bilden die aktiven Mitglieder des Online-Access-Panels der Respondi AG. Dieses Panel umfasste 2009 ca. 65.000 Personen. Ihre Rekrutierung erfolgte online über Meinungsportale, On-Site-Befragungen und Suchmaschinen sowie zu einem geringen Teil auch offline per Telefon. Die Stichprobe für die Online-Umfrage wurde als Quotenstichprobe mit den Quotierungsmerkmalen Geschlecht, Bildung, Alter realisiert, wobei Befragte in mehreren Wellen zur Erfüllung der Quotenvorgaben eingeladen wurden. Des Weiteren wurden die Quotierungsmerkmale nachträglich an Sollwerte des Mikrozensus und an die OnlinePopulation (ab 18 Jahren mit deutscher Staatsangehörigkeit) angepasst (Referenzgrundlage: Onliner-Atlas 2008). Von den 3318 Personen, die von Respondi eingeladen wurden, hat knapp die Hälfte (1634) das Interview begonnen. Von diesen haben 213 Personen das Interview abgebrochen, weitere 268 wurden abgewiesen, da die Quotenvorgaben bereits erfüllt waren. Damit stehen 1153 komplettierte Interviews zur Analyse zur Verfügung. ${ }^{9}$

8 Es handelt sich dabei um folgende Studie: GLES 1006 - Komponente X/8 - Online-Tracking 6: Wahlkampf. Die Daten wurden von GESIS - Leibniz-Institut für Sozialwissenschaften aufbereitet und dokumentiert und für die Analyse zugänglich gemacht. Sie wurden erhoben unter der Federführung von Prof. Dr. Hans Rattinger (Universität Mannheim), Prof. Dr. Sigrid Roßteutscher (Universität Frankfurt), Prof. Dr. Rüdiger Schmitt-Beck (Universität Mannheim) und PD Dr. Bernhard Weßels (Wissenschaftszentrum Berlin). Weder die genannten Personen noch Institute tragen Verantwortung für die Analyse oder Interpretation der Daten in diesem Beitrag.

9 Zum methodischen Vorgehen der Stichprobenziehung und Gewichtung sei auf den Methodenbericht der entsprechenden Studie verwiesen, der nach Registrierung unter nachstehendem Link zusammen mit den Daten abrufbar ist: http://www.gesis.org/wahlen/gles/daten-und-dokumente/ daten/ (Stand: 18.03. 2012). 
Aufgrund der umfangreichen Grundgesamtheit und der quotierten Stichprobe erhebt die Studie Anspruch auf Online-Repräsentativität und bietet im Vergleich zur Datenqualität anderer in der VAA-Forschung vorgenommener Erhebungen einige Vorteile. Zunächst werden durch den Rückgriff auf ein Online-Panel sonst schwer zugängliche Gruppen wie Niedriggebildete oder ältere Menschen in entsprechender Proportion berücksichtigt, was z. B. bei online-basierten Umfragen, die auf Selbst-Selektion beruhen (vgl. Walgrave et al. 2006, S. 5), nicht gewährleistet ist. Zudem können mit den vorliegenden Daten Vergleichsgruppen gebildet werden - ein Vorzug gegenüber Datensätzen, die auf Exit-Surveys beruhen und damit nur Nutzer der VAAs erfassen.

Nach Darstellung dieser Vorzüge muss allerdings einschränkend hinzugefügt werden, dass es sich bei dem Sample nicht um eine Zufallsstichprobe aller Wahlberechtigten handelt, sondern um eine Quotenstichprobe der wahlberechtigten Online-Nutzer in Deutschland. Anders als VAA-Studien, die mit repräsentativen Befragungen auf Grundlage von Zufallsstichproben arbeiten (Mykkänen u. Moring 2006; Nuytemans et al. 2010; Ruusuvirta u. Rosema 2009), können die Befunde unserer Analyse nicht auf die Gesamtbevölkerung übertragen werden. Da das Quoten-Verfahren keine Zufallsauswahl darstellt, ist auch die Anwendung von Inferenzstatistiken diskutabel (vgl. Schnell et al 2008, S. 303). Durch die hohen Qualitätsstandards der Respondi AG bei der Panelrekrutierung und Panelpflege erscheint es uns allerdings berechtigt anzunehmen, dass die Stichprobe einer Zufallsauswahl der wahlberechtigten Online-Population angenähert ist, sodass die Nutzung von Inferenzstatistiken und die Übertragung der Ergebnisse auf die wahlberechtigte Online-Population in Deutschland zulässig ist. ${ }^{10}$

Bei der Analyse arbeiten wir mit Vergleichsgruppen: Anhand der Frage „Haben Sie den Wahl-O-Mat genutzt?" können mit den Daten der GLES trennscharfe, überlappungsfreie Gruppen mit folgenden Merkmalen gebildet werden: 1) Personen, die den Wahl-O-Mat kennen und gespielt haben (Kenner/Nutzer), 2) Personen, die den Wahl-O-Mat kennen, aber nicht gespielt haben (Kenner/Nicht-Nutzer), und 3) Personen, die den Wahl-O-Mat weder kennen, noch gespielt haben (Nicht-Kenner/Nicht-Nutzer). Diese Vergleichsgruppenbildung erlaubt die Ermittlung eines VAA-Effekts auf die Wahlbeteiligungsabsicht der Gruppe der Nutzer in Abgrenzung zu den anderen Gruppen, eine Vorgehensweise, die bislang in dieser Form selbst bei repräsentativen Studien zu VAAs noch nicht realisiert worden ist. Wir gehen davon aus, dass sich innerhalb der Gruppe der Nicht-Nutzer diejenigen, die den Wahl-O-Mat kennen, von denen, die den Wahl-O-Mat nicht kennen, deutlich unterscheiden.

Gewichtet nach Onlinern zeigt die Häufigkeitsverteilung der Variable, die nach der Wahl-O-Mat-Nutzung fragt, dass die deutsche VAA bei den Befragten einen hohen Bekanntheitsgrad genießt: Von den 1146 Personen, die zu dieser Frage Angaben machten, geben 38,5 Prozent an, den Wahl-O-Mat genutzt zu haben, 45,1 Prozent kennen den Wahl-O-Mat, haben ihn aber zur Bundestagswahl 2009 nicht verwendet, und nur 16,4 Prozent sagen, dass ihnen das Tool nicht bekannt sei. In

10 Vgl. zu diesem Vorgehen auch Schoen 2010. 
einer repräsentativen Befragung anlässlich der Wahl des niederländischen Parlaments 2006 berichten Ruusuvirta und Rosema (2009, S. 12) von einem ebenfalls hohen Bekanntheitsgrad des Stemwijzers, der sich allerdings auf das gesamte Elektorat und nicht nur auf die Internet-Gemeinde bezieht: 61 Prozent der Wahlberechtigten kennen die niederländische VAA, wobei 38 Prozent von diesen „Kennern" das Tool gespielt haben.

\subsection{Soziodemografische Merkmale und politische Einstellungen der Vergleichsgruppen}

Studien zu VAAs haben gezeigt, dass sich die soziodemografische Zusammensetzung sowie die politischen Einstellungen der VAA-User von der Gesamtheit der Wahlberechtigten, aber auch von der Internet-Population unterscheiden. Der typische Nutzer wird charakterisiert als männlich, jung, formal gut gebildet und politisch interessiert. ${ }^{11} \mathrm{Da}$ die Angaben für die deutsche VAA-Nutzerschaft bislang nur auf Exit-Befragungen beruhen, überprüfen wir zunächst diese Charakterisierung mithilfe der online-repräsentativen GLES-Daten, aufgeschlüsselt nach den drei Vergleichsgruppen. Zusätzlich beziehen wir für eine weitere Beschreibung der Nutzer folgende Variablen ein: (1) Demokratiezufriedenheit, (2) Interesse am Wahlkampf und (3) Wichtigkeit des Ausgangs der Wahl für den Befragten. Mit Blick auf diese Variablen liegen Erkenntnisse vor, dass eine jeweils höhere Ausprägung die Wahlbeteiligungsabsicht signifikant steigert (Westle u. Niedermayer 2009; Johann 2009; Faas 2010; Steinbrecher u. Rattinger 2011). Diejenigen Variablen, die sich als relevant für die Wahl-O-Mat-Nutzung erweisen, berücksichtigen wir in unserer anschließenden Analyse zur Wahlbeteiligung, indem wir sie bei der Ermittlung eines etwaigen VAA-Effekts kontrollieren.

Tabelle 1 weist die bivariaten Zusammenhänge zwischen den soziodemografischen Merkmalen und politischen Einstellungen als jeweils unabhängige Variable und der Wahl-O-Mat-Nutzung als abhängige Variable aus.

11 Vgl. De Rosa 2010; Ladner u. Pianzola 2010; Ladner et al. 2008; Marschall u. Schmidt 2010; Mykkänen u. Moring 2006; Wall et al. 2009. 
Tabelle 1: Zusammenhänge zwischen Wahl-O-Mat-Nutzung und soziodemografischen Merkmale sowie politischen Einstellungen (Zeilenprozente)

\begin{tabular}{|c|c|c|c|}
\hline & $\begin{array}{l}\text { Kenner/Nutzer } \\
\text { in } \%(N)\end{array}$ & $\begin{array}{c}\text { Kenner/ } \\
\text { Nicht-Nutzer } \\
\text { in } \%(\mathrm{~N}) \\
\end{array}$ & $\begin{array}{c}\text { Nicht-Kenner/ } \\
\text { Nicht-Nutzer } \\
\text { in \% (N) }\end{array}$ \\
\hline Befragte & $38,5(441)$ & $45,1(517)$ & $16,4(188)$ \\
\hline \multicolumn{4}{|l|}{ Soziodemografische Merkmale } \\
\hline \multicolumn{4}{|l|}{ Geschlecht } \\
\hline männlich & $42,5(248)$ & $45,7(267)$ & $11,8(69)$ \\
\hline weiblich & $33,9(190)$ & $44,9(252)$ & $21,2(119)$ \\
\hline Cramers V & & & $0,135 * *$ \\
\hline \multicolumn{4}{|l|}{ Höchster Bildungsabschluss } \\
\hline $\begin{array}{l}\text { kein Abschluss oder } \\
\text { Hauptschulabschluss }\end{array}$ & $25,4(90)$ & $51,1(181)$ & $23,4(83)$ \\
\hline Mittlere Reife & $34,9(158)$ & $46,6(211)$ & $18,5(84)$ \\
\hline FH-Reife oder Abitur & $55,2(171)$ & $38,4(119)$ & $6,5(20)$ \\
\hline Cramers V & & & $0,185 * *$ \\
\hline \multicolumn{4}{|l|}{ Alter } \\
\hline $18-24$ & $66,5(127)$ & $25,7(49)$ & $7,9(15)$ \\
\hline $25-34$ & $44,1(83)$ & $44,1(83)$ & $11,7(22)$ \\
\hline $35-44$ & $35,1(85)$ & $47,1(114)$ & $17,8(43)$ \\
\hline $45-60$ & $31,0(107)$ & $50,4(174)$ & $18,6(64)$ \\
\hline $61+$ & $19,0(33)$ & $55,7(97)$ & $25,3(44)$ \\
\hline Cramers V & & & $0,217 * *$ \\
\hline \multicolumn{4}{|l|}{ Politische Einstellungen } \\
\hline \multicolumn{4}{|l|}{ Politisches Interesse } \\
\hline überhaupt nicht oder schwach & $21,3(42)$ & $45,2(89)$ & $33,5(66)$ \\
\hline mittelmäßig & $34,4(140)$ & $47,2(192)$ & $18,4(75)$ \\
\hline stark oder sehr stark & $47,3(256)$ & $44,0(238)$ & $8,7(47)$ \\
\hline Cramers V & & & $0,192 * *$ \\
\hline \multicolumn{4}{|l|}{ Demokratiezufriedenheit } \\
\hline sehr und ziemlich unzufrieden & $30,8(82)$ & 47,7 (127) & $21,4(57)$ \\
\hline teils teils & $37,0(191)$ & $45,9(237)$ & $17,1(88)$ \\
\hline ziemlich und sehr zufrieden & $45,3(162)$ & $42,7(153)$ & $12,0(43)$ \\
\hline Cramers V & & & $0,089 * *$ \\
\hline \multicolumn{4}{|l|}{ Interesse am Wahlkampf } \\
\hline überhaupt nicht oder schwach & $23,8(49)$ & $46,1(95)$ & $30,1(62)$ \\
\hline mittelmäßig & $34,1(133)$ & $49,2(192)$ & $16,7(65)$ \\
\hline stark oder sehr stark & $46,4(253)$ & $42,4(231)$ & $11,2(61)$ \\
\hline Cramers V & & & $0,164 * *$ \\
\hline
\end{tabular}




\begin{tabular}{lccc} 
& $\begin{array}{c}\text { Kenner/Nutzer } \\
\text { in \% (N) }\end{array}$ & $\begin{array}{c}\text { Kenner/ } \\
\text { Nicht-Nutzer } \\
\text { in \% (N) }\end{array}$ & $\begin{array}{c}\text { Nicht-Kenner/ } \\
\text { Nicht-Nutzer } \\
\text { in \% (N) }\end{array}$ \\
\hline Wichtigkeit des Ausgangs der Wahl & & & \\
überhaupt nicht oder wenig wichtig & $21,1(20)$ & $43,2(41)$ & $35,8(34)$ \\
teils teils & $27,4(63)$ & $47,0(108)$ & $25,7(59)$ \\
wichtig oder sehr wichtig & $43,7(358)$ & $44,8(367)$ & $11,6(95)$ \\
Cramers V & & & $0,170^{* *}$
\end{tabular}

Anmerkungen: *: p<0,05 ; **: p<,0,01. Quelle: eigene Berechnungen. Datengrundlage: GLES 1006 - Komponente X/8 - Online-Tracking 6: Wahlkampf (gewichtet nach Onlinern).

Die Ergebnisse zeigen, dass die ausgewählten Variablen einen schwachen bis mittelstarken Einfluss auf die Nutzung und Bekanntheit des Wahl-O-Mat haben. Unter den weiblichen Befragten ist der Anteil derjenigen, die den Wahl-O-Mat nicht kennen, fast doppelt so groß wie unter den männlichen Personen, wobei das Geschlecht unter den soziodemografischen Merkmalen den schwächsten Einfluss ausweist. Etwas stärker ist der Einfluss des Bildungsabschlusses: Knapp mehr als die Hälfte der Befragten mit FH-Reife oder Abitur haben den Wahl-O-Mat zur Bundestagswahl 2009 genutzt, während nur 6,5 Prozent der Personen mit diesem Bildungsabschluss das Tool nicht kennen. Zugleich ist der Anteil der Kenner/NichtNutzer auf jedem Bildungsniveau recht hoch. Den stärksten Effekt hat das Alter. Unter den 18- bis 24-jährigen finden sich 66,5 Prozent Nutzer/Kenner, aber nur 7,9 Prozent Nicht-Nutzer/Nicht-Kenner. In der höchsten Alterskategorie haben hingegen nur 19 Prozent der Befragten das Tool gespielt, wobei der Anteil derjenigen, die die deutsche VAA zumindest kennen, mit 55,7 Prozent sehr hoch ist.

Bei den politischen Einstellungsvariablen hat „Demokratiezufriedenheit“ den schwächsten Einfluss auf die Wahl-O-Mat-Nutzung, wobei die Gruppe der Kenner/Nicht-Nutzer in jeder Zufriedenheitsausprägung mit jeweils über 40 Prozent recht stark vertreten ist. Einen deutlicheren Einfluss hat das politische Interesse: In der Gruppe der politisch stark oder sehr stark interessierten Internet-Nutzer liegt der Anteil derer, die vom Wahl-O-Mat noch nie gehört haben, bei gerade einmal 8,7 Prozent; auf die Kenner/Nicht-Nutzer entfallen hingegen 44 Prozent und auf die Kenner/Nutzer 47,3 Prozent. Ähnliche Verteilungen zeigen sich bei den Variablen „Interesse am Wahlkampf“ und „Wichtigkeit des Ausgangs der Wahl“. In den jeweils höchsten Ausprägungen finden sich vergleichsweise viele Kenner und/oder Nutzer und wenige Nicht-Kenner/Nicht-Nutzer.

Die Ergebnisse der Kreuztabellierungen bestätigen für jede unabhängige Variable die auf der Grundlage der vorliegenden Exit-Surveys ermittelte Charakterisierung der Wahl-O-Mat-Nutzer, wobei die Variablen „Demokratiezufriedenheit“, „Interesse am Wahlkampf“ und „Wichtigkeit des Ausgangs der Wahl“, die bislang nicht in Exit-Studien erhoben wurden, wie auch das politische Interesse das gleiche Muster aufweisen. Allerdings gelten diese Zuschreibungen nur für den Fall, dass alleine die Gruppe der Kenner/Nutzer mit der Gruppe der Nicht-Kenner/NichtNutzer verglichen wird. Beim Vergleich der Kenner/Nicht-Nutzer mit den Kennern/Nutzern gestaltet sich das Bild etwas differenzierter: Bildung und Alter wei- 
sen den erwarteten Zusammenhang auf. Ausprägungen wie die wahrgenommene hohe persönliche Wichtigkeit des Wahlausgangs, eine hohe Demokratiezufriedenheit sowie starkes politisches Interesse und Interesse am Wahlkampf treffen aber auch für diejenigen Personen zu, die das Tool kennen, aber nicht spielen. Insofern sind sich die Nutzer und die Kenner/Nicht-Nutzer in einigen Hinsichten ähnlicher, als es die beiden Untergruppen der Nicht-Nutzer (Kenner vs. Nicht-Kenner) sind, was für die vorgenommene Einteilung in drei Vergleichsgruppen spricht.

Insgesamt stellen soziodemografische Variablen sowie politische Einstellungen (darunter in abgeschwächter Form die Demokratiezufriedenheit) Größen dar, welche die Wahl-O-Mat-Nutzung beeinflussen. Um den Effekt der VAA auf die Bereitschaft zur Wahl zu gehen ohne eine Verzerrung durch die typische Zusammensetzung der Nutzerschaft und kontrolliert für relevante Variablen zur Erklärung der Wahlbeteiligungsabsicht zu ermitteln, werden diese Merkmale - neben weiteren Drittvariablen - in den folgenden Analysen kontrolliert.

\subsection{Effekt der Wahl-O-Mat-Nutzung auf die Wahlbeteiligung}

Um die Wirkung der Wahl-O-Mat-Nutzung auf die Wahlbeteiligung zu schätzen, verwenden wir binär logistische Regressionen (vgl. Backhaus et al. 2003, S. 426487; Best u. Wolf 2010; Menard 2002; Sharma 1996, S. 328-332) mit der Wahlbeteiligungsabsicht als dichotomer abhängiger Variable. ${ }^{12}$ Tabelle 2 weist die Odds Ratios sowie die Standardfehler aus. Die Odds Ratios werden wie folgt in unserem Modell interpretiert: Werte zwischen 0 und 1 bedeuten, dass sich die Eintrittswahrscheinlichkeit der beabsichtigten Wahlteilnahme im Vergleich zur Referenzkategorie verringert. Werte über 1 bedeuten eine Erhöhung der Eintrittswahrscheinlichkeit (vgl. dazu auch allgemein Diaz-Bone u. Künemund 2003, S. 9-10; Fromm 2005, S. 24). Des Weiteren wird ein globales Gütemaß kalkuliert. Im Gegensatz zum $\mathrm{R}^{2}$ der linearen Regression existiert für die Interpretation der Modellgüte in der logistischen Regression eine Vielzahl verschiedener Pseudo- ${ }^{2}$-Maße und es herrscht Uneinigkeit über die beste Maßzahl (vgl. Cameron u. Windmeijer 1997). Da die Anzahl der unabhängigen Variablen zwischen unseren Modellen variiert, verwenden wir im Folgenden das für die Anzahl der verwendeten Prädiktoren korrigierte McFaddens Pseudo-R². Gemäß Diaz-Bone (2006, S. 244) indizieren dabei bereits Werte über 0,2 einen guten Modellfit. Aufgrund des unsererseits vermuteten Effekts der Wahl-O-Mat-Nutzung auf die Wahlbeteiligungsabsicht sollten sich signifikante Werte über 1 für die Wahl-O-Mat-Nutzung im Modell ergeben.

Als unabhängige Variablen für die Erklärung der Wahlbeteiligungsabsicht führen wir zunächst die soziodemografischen Variablen als Block ein (Modell 1). In Modell 2 werden zusätzlich politische Einstellungsvariablen aufgenommen, die in der Lage sind, die Wahlbeteiligungsabsicht zu erklären, und wie dargestellt einen Einfluss auf das Kennen und Nutzen des Wahl-O-Mat haben (s. Abschnitt 3.2).

12 Es handelt sich dabei um die Variable a07, welche die Wahlbeteiligungsabsicht abfragt. Aus der Analyse wurden Wähler, die bereits per Briefwahl gewählt haben, ausgeschlossen. Zum anderen wurde die Variable so rekodiert, dass $0=$ „nicht sichere Wahlteilnahme“ und $1=$ „, sichere Wahlteilnahme" darstellt. 
Um eine möglichst konservative Überprüfung des Wahl-O-Mat-Effektes zu gewährleisten, integrieren wir in Modell 3 neben der Wahl-O-Mat-Nutzung weitere kurzfristige Faktoren, die sich positiv auf die Wahlbeteiligungsabsicht wie auch auf die Nutzung des Wahl-O-Mat auswirken können. Zu diesen gehören das Vorhandensein einer Kanzlerpräferenz sowie weitere Formen der politischen Kommunikation. Mit der Berücksichtigung der Kanzlerpräferenz nehmen wir einen kandidatenbezogenen Indikator auf, der gleichfalls Einfluss auf die Wahlbeteiligungsabsicht nehmen kann (Gabriel u. Neller 2005; Gabriel et al. 2009; Schoen 2007, 2011; kritisch Wagner u. Weßels 2012). Die Einbindung der Nutzung weiterer möglicher Kommunikationseinflüsse in unser Modell trägt der Überlegung Rechnung, dass die Nutzung des Wahl-O-Mat in einen Komplex politischer Kommunikation eingebettet ist (vgl. Brettschneider 2005), der den Wahl-O-Mat-Effekt überdecken könnte. Das Modell berücksichtigt zwei „push“-Formen politischer Kommunikation (Wahrnehmung des TV-Duells, Erhalt von Parteiinformationen) sowie neben der Wahl-O-Mat-Nutzung eine weitere „pull“-Form politischer Kommunikation (Lesen von Wahlprogrammen). ${ }^{13} \mathrm{Im}$ Modell 4 wird schließlich überprüft, ob der postulierte Zusammenhang bestehen bleibt, wenn in einer alternativen Kodierung die Nicht-Kenner und die Kenner/Nicht-Nutzer zusammengefasst als Referenzkategorie modelliert und gegen die Gruppe der Wahl-O-Mat-Nutzer getestet werden, sodass ausschließlich zwischen Nutzern des Tools und den übrigen Befragten unterschieden wird. In allen Modellen werden jeweils die niedrigsten Ausprägungen der unabhängigen Variablen als Referenzkategorie modelliert.

\section{Tabelle 2: Logistische Regressionen zur Erklärung der Wahlbeteiligungsabsicht - Odds Ratios}

\begin{tabular}{|c|c|c|c|c|c|c|c|c|}
\hline \multirow{2}{*}{\multicolumn{3}{|c|}{$\begin{array}{c}\text { Modell } 1 \\
\text { Geschlecht (Referenz: männlich) }\end{array}$}} & \multicolumn{2}{|c|}{ Modell 2} & \multicolumn{2}{|c|}{ Modell 3} & \multicolumn{2}{|c|}{ Modell 4} \\
\hline & & & & & & & & \\
\hline weiblich & 0,745 & $(0,192)$ & 1,061 & $(0,231)$ & 1,017 & $(0,237)$ & 1,023 & $(0,236)$ \\
\hline \multicolumn{9}{|c|}{$\begin{array}{l}\text { Höchster Bildungsabschluss } \\
\text { (Referenz: kein Abschluss oder Hauptschulabschluss) }\end{array}$} \\
\hline Mittlere Reife & $1,821 * *$ & $(0,216)$ & 1,135 & $(0,260)$ & 1,056 & $(0,268)$ & 1,049 & $(0,267)$ \\
\hline Abitur & $3,646 * *$ & $(0,284)$ & 1,683 & $(0,329)$ & 1,383 & $(0,351)$ & 1,363 & $(0,349)$ \\
\hline \multicolumn{9}{|c|}{ Alter (Referenz: 18-24) } \\
\hline $25-34$ & 0,858 & $(0,329)$ & 1,121 & $(0,388)$ & 1,083 & $(0,411)$ & 1,078 & $(0,410)$ \\
\hline $35-44$ & 0,931 & $(0,316)$ & 0,720 & $(0,372)$ & 0,812 & $(0,405)$ & 0,814 & $(0,404)$ \\
\hline $45-60$ & 1,648 & $(0,329)$ & 1,466 & $(0,378)$ & 1,668 & $(0,422)$ & 1,679 & $(0,422)$ \\
\hline $61+$ & 1,324 & $(0,369)$ & 0,903 & $(0,436)$ & 1,057 & $(0,470)$ & 1,066 & $(0,469)$ \\
\hline \multicolumn{9}{|c|}{$\begin{array}{l}\text { Politisches Interesse (Referenz: } \\
\text { schwach oder überhaupt nicht) }\end{array}$} \\
\hline \multirow{2}{*}{\multicolumn{3}{|c|}{$\begin{array}{l}\text { mittelmäßig } \\
\text { stark oder } \\
\text { sehr stark }\end{array}$}} & 1,607 & $(0,311)$ & 1,374 & $(0,327)$ & 1,378 & $(0,327)$ \\
\hline & & & $2,690 *$ & $(0,388)$ & $2,266^{*}$ & $(0,414)$ & $2,249 *$ & $(0,414)$ \\
\hline
\end{tabular}

13 Zur Unterscheidung zwischen „push“- und „pull“-Formen politischer Kommunikation siehe Schulz 2009, S. 227. 


\begin{tabular}{|c|c|c|c|}
\hline \multicolumn{4}{|c|}{$\begin{array}{l}\text { Demokratiezufriedenheit (Refe- } \\
\text { renz: ziemlich und sehr unzufrieden }\end{array}$} \\
\hline teils teils & $1,651 \quad(0,263)$ & $1,675 \quad(0,282)$ & $1,680 \quad(0,282)$ \\
\hline $\begin{array}{l}\text { ziemlich und } \\
\text { sehr zufrieden }\end{array}$ & $2,621 *(0,325)$ & $2,703 * *(0,349)$ & $2,692 * *(0,349)$ \\
\hline \multicolumn{4}{|c|}{$\begin{array}{l}\text { Interesse am Wahlkampf (Referenz: } \\
\text { überhaupt nicht oder schwach }\end{array}$} \\
\hline mittelmäßig & $1,027 \quad(0,314)$ & $0,982 \quad(0,332)$ & $0,977 \quad(0,332)$ \\
\hline $\begin{array}{l}\text { stark oder sehr } \\
\text { stark }\end{array}$ & $1,601 \quad(0,415)$ & $1,377 \quad(0,453)$ & $1,377 \quad(0,453)$ \\
\hline \multicolumn{4}{|c|}{$\begin{array}{l}\text { Wichtigkeit der Ausgang der Wahl } \\
\text { (Referenz: überhaupt nicht oder wenig wichtig) }\end{array}$} \\
\hline teils teils & $2,007 \quad(0,380)$ & $2,210 *(0,395)$ & $2,209 * \quad(0,395)$ \\
\hline $\begin{array}{l}\text { wichtig oder } \\
\text { sehr wichtig }\end{array}$ & $11,067 *(0,395)$ & $10,090 *(0,411)$ & $10,045 *(0,411)$ \\
\hline \multicolumn{4}{|c|}{ Kanzlerpräferenz (Referenz: keine) } \\
\hline Merkel & & $1,009 \quad(0,287)$ & $1,009 \quad(0,287)$ \\
\hline Steinmeier & & $0,985 \quad(0,315)$ & $0,980 \quad(0,315)$ \\
\hline \multicolumn{4}{|c|}{ TV-Duell (Referenz: nicht gesehen) } \\
\hline $\begin{array}{l}\text { wenig oder nicht } \\
\text { aufmerksam }\end{array}$ & & $2,113 \quad(0,432)$ & $2,094 \quad(0,431)$ \\
\hline $\begin{array}{l}\text { aufmerksam oder } \\
\text { sehr aufmerksam }\end{array}$ & & $1,297 \quad(0,300)$ & $1,290 \quad(0,299)$ \\
\hline \multicolumn{4}{|l|}{$\begin{array}{l}\text { Wahlprogramm gelesen } \\
\text { (Referenz: nein) }\end{array}$} \\
\hline ja & & $1,362 \quad(0,310)$ & $1,367 \quad(0,310)$ \\
\hline \multicolumn{4}{|c|}{$\begin{array}{l}\text { Informationen von Parteien } \\
\text { erhalten (Referenz: nein) }\end{array}$} \\
\hline ja & & $2,031 * *(0,240)$ & $1,997 * *(0,237)$ \\
\hline \multicolumn{4}{|c|}{$\begin{array}{l}\text { Wahl-O-Mat-Nutzung (Referenz: } \\
\text { Nicht-Kenner /Nicht-Nutzer) }\end{array}$} \\
\hline $\begin{array}{l}\text { Kenner/Nicht- } \\
\text { Nutzer }\end{array}$ & & $0,878 \quad(0,309)$ & \\
\hline Kenner/Nutzer & & $2,196 *(0,378)$ & \\
\hline \multicolumn{4}{|c|}{$\begin{array}{l}\text { Wahl-O-Mat-Nutzung binär } \\
\text { (Referenz: nein) }\end{array}$} \\
\hline $\mathrm{Ja}$ & & & $2,443 * *(0,289)$ \\
\hline $\begin{array}{l}\text { Korr. Pseudo-R } R^{2} \\
\text { (McFadden) }\end{array}$ & 0,23 & 0,25 & 0,25 \\
\hline 782 & 782 & 782 & 782 \\
\hline
\end{tabular}

Anmerkungen: ${ }^{*}: \mathrm{p}<0,05 ;{ }^{* *}: \mathrm{p}<, 0,01$; Standardfehler in Klammern. Quelle: eigene Berechnungen. Datengrundlage: GLES 1006 - Komponente X/8 - Online-Tracking 6: Wahlkampf (gewichtet nach Onlinern). 
Die Ergebnisse für das Modell 1, welches nur die soziodemografischen Variablen beinhaltet, weisen aus, dass alleine der Bildungsabschluss einen Einfluss auf die Wahlbeteiligungsabsicht hat, nicht aber das Alter und Geschlecht. So steigt mit einem höheren Bildungsabschluss die Bereitschaft, zur Wahl zu gehen. Jedoch signalisiert Pseudo-R ${ }^{2}$ einen nicht akzeptablen Modellfit für Modell 1.

Mit Hinzufügen der politischen Einstellungsvariablen verlieren alle soziodemografischen Variablen ihren signifikanten Einfluss auf die Wahlbeteiligungsabsicht (Modell 2). Starkes politisches Interesse, hohe Demokratiezufriedenheit und die Einschätzung, dass der Ausgang der Wahl für die Befragten persönlich wichtig oder sehr wichtig ist, erhöhen die Absicht, zur Wahl zu gehen, im Vergleich zu den jeweils niedrigsten Ausprägungen dieser Variablen. Vor allem Personen, denen das Ergebnis der Wahl wichtig oder sehr wichtig ist, haben eine rund zehnmal höhere Chance, eine positive Wahlbeteiligungsabsicht aufzuweisen, als diejenigen, die bekunden, dass für sie der Wahlausgang nicht wichtig sei - ein Befund, der angesichts der inhaltlichen Nähe dieser unabhängigen Variable zur abhängigen Variable wenig überrascht. Die Stärke des Interesses am Wahlkampf hat hingegen - bei Kontrolle der anderen politischen Einstellungsvariablen - keinen signifikanten Einfluss.

Mit Integration der politischen Einstellungsvariablen erhöht sich der Modellfit erheblich auf den Wert von 0,23. Diejenigen ausgewählten politischen Einstellungsvariablen, die signifikant sind, erbringen somit eine gute Erklärungsleistung für die Wahlbeteiligungsabsicht. Diese Beobachtung korrespondiert mit Befunden anderer Studien, die gleichfalls einen Effekt dieser Variablen auf die Wahlbeteiligung feststellen (Steinbrecher u. Rattinger 2011, S. 82-89; Westle u. Niedermayer 2009, S. 25; Johann 2009; Faas 2010).

Im dritten Modell werden die Kanzlerpräferenz, politische Kommunikationsvariablen und die Wahl-O-Mat-Nutzung in die Analyse einbezogen. Von diesen Variablen hat neben der Wahl-O-Mat-Nutzung nur der Erhalt von Informationen durch Parteien einen signifikant positiven Einfluss auf die Wahlbeteiligungsabsicht. Kontrolliert für die Variablen aus Modell 3 hat das bloße Kennen des WahlO-Mat keinen signifikanten Einfluss, jedoch macht eine Wahl-O-Mat-Nutzung die Wahlteilnahme wahrscheinlicher. So ist die relative Chance, eine positive Wahlbeteiligungsabsicht vorzuweisen, bei den Wahl-O-Mat-Nutzern rund 2,2mal höher als im Vergleich zur Referenzgruppe der Nicht-Kenner/Nicht-Nutzer; dieser Effekt ist auf dem 5\%-Niveau signifikant. Im Modell 4, in dem ohne Einfügung weiterer Variablen dichotom nach Wahl-O-Mat-Nutzung bzw. Nicht-Nutzung unterschieden wird, ist die relative Chance, eine positive Wahlbeteiligungsabsicht zu haben, bei den Nutzern des Tools sogar rund 2,4-mal höher gegenüber den übrigen Befragten und auf dem 1\%-Niveau signifikant. Die Größe der Standardfehler legt zudem den Schluss nahe, dass die Odds Ratios in jedem Modell hinreichend präzise geschätzt worden sind.

Eine weitere Möglichkeit zur Illustration des Wahl-O-Mat-Effekts auf der Basis der durchgeführten Datenauswertung ist die Angabe der Veränderung der Wahrscheinlichkeiten für eine sichere Wahlabsicht durch die Nutzung des Wahl-O-Mat bei Konstanthaltung der übrigen unabhängigen Variablen in einer bestimmten Kombination von Ausprägungen - also für „typische“ Personengruppen. Grundla- 
ge für diese Berechnungen sind die Werte aus Modell 4. Dabei unterscheiden sich die Ausgangswahrscheinlichkeiten für eine sichere Wahlbeteiligungsabsicht je nach Personengruppe deutlich: So liegt beispielsweise die Wahrscheinlichkeit für eine Person, die bei allen unabhängigen Variablen in der (niedrigen) Referenzkategorie von Tabelle 3 verortet ist (d. h. männlich, niedriges Bildungsniveau, jüngste Alterskategorie, nicht politisch interessiert etc.), ohne Wahl-O-Mat-Nutzung mit 9,5 Prozent sehr niedrig. Eine Person mit den gleichen Charakteristika, die aber den Wahl-O-Mat gespielt hat, weist hingegen eine 20,3-prozentige Wahrscheinlichkeit auf, sicher an der Wahl teilzunehmen. Befragte wiederum, die bei allen unabhängigen Variablen in den jeweils höchsten Ausprägungen geantwortet haben, aber die deutsche VAA nicht nutzten, haben bereits eine Wahrscheinlichkeit von 97,9 Prozent, sicher an der Wahl teilzunehmen; eine Nutzung des Tools erhöht diese Wahrscheinlichkeit auf 99,1 Prozent. Neben diesen extremen Beispielen kann der Effekt des Wahl-O-Mat auch für eine typische Person im Datensatz berechnet werden, deren Ausprägungen auf der Modal- bzw. Mediankategorie aller Variablen liegt. ${ }^{14}$ Diese Person weist ohne die Nutzung des Wahl-O-Mat eine Wahrscheinlichkeit von 80,3 Prozent auf, eine sichere Wahlbeteiligungsabsicht zu haben; mit der Nutzung des Wahl-O-Mat erhöht sich dieser Wert auf 90,9 Prozent.

Der beachtliche Mobilisierungseffekt des Wahl-O-Mat ist somit kein Artefakt einer spezifischen soziodemografischen Zusammensetzung der Nutzerschaft, sondern es besteht gemäß unseren Modellen ein unabhängiger VAA-Effekt, der sich auch dann noch positiv auf die Bereitschaft zur Wahl zu gehen auszuwirken scheint, wenn für eine Vielzahl relevanter Drittvariablen zur Erklärung der Wahlbeteiligungsabsicht kontrolliert wird.

\section{Fazit}

Die Verbreitung von Voting Advice Applications und ihre Popularität werfen die Frage auf, ob und inwiefern die Nutzung dieser Online-Tools Auswirkungen auf die politische Partizipation, insbesondere auf die Wahlbeteiligung hat. Eine unter Rückgriff auf verschiedene theoretische Ansätze vorgenommene Plausibilisierung sowie erste Befunde aus der europäischen VAA-Forschung legen die Vermutung nahe, dass die Nutzung von Voting Advice Applications in der Tat einen positiven Effekt auf die Wahlbereitschaft hat. Diese Vermutung haben wir anhand der deutschen VAA, dem Wahl-O-Mat, auf der Basis eines Datensatzes des GLES-Projektes zur Bundestagswahl 2009 mittels logistischer Regressionsanalysen überprüft. Die Erwartung, dass VAAs eine Auswirkung auf die politische Beteiligung haben, ist dabei bestätigt worden. So hat die Wahl-O-Mat-Nutzung einen messbaren, positiven Effekt auf die Absicht zur Wahl zu gehen - ein Befund, der im Einklang mit internationalen Forschungsergebnissen zu vergleichbaren Tools steht (z. B. De

14 Dieser typische Befragte ist männlich, hat einen mittleren Bildungsabschluss, ist 35 bis 44 Jahre alt, hat ein mittleres politisches Interesse, eine mittlere Demokratiezufriedenheit und mittleres Interesse am Wahlkampf, er schätzt den Ausgang der Wahl als wichtig oder sehr wichtig ein, präferiert Merkel als Kanzlerin, hat das TV-Duell nicht gesehen, kein Wahlprogramm gelesen, aber von Parteien Informationen erhalten. 
Rosa 2010; Nuytemans et al. 2010; Mykkänen u. Moring 2006; Ruusuvirta u. Rosema 2009). Dieser Effekt bleibt - wie wir zeigen konnten - auch dann noch bestehen, wenn für relevante demografische Merkmale, politische Einstellungsvariablen, die Kandidatenorientierung und andere Formen politischer Kommunikation kontrolliert wird.

Welche Rückschlüsse können wir auf der Grundlage dieser Ergebnisse auf die Kontroverse zwischen Normalisierungs- und Mobilisierungsthese ziehen? Mit der deutschen VAA haben wir eine Online-Applikation analysiert, die offensichtlich in der Lage ist, Personen zur Teilnahme an Wahlen zu mobilisieren - und dies unabhängig von soziodemografischen Merkmalen und anderen Charakteristika, insbesondere politischen Einstellungen, welche die Wahlbeteiligungsabsicht ansonsten zu erklären vermögen. Die Wirkung des Tools stellt einen „Medieneffekt“ (Bryant u. Oliver 2009) der bei den ohnehin Mobilisierten greift, aber notabene auch bei Personen, die als politikfern eingestuft werden können. Insofern unterstützen unsere Ergebnisse die Mobilisierungsthese, da die Wirkung nicht auf bereits Mobilisierte beschränkt bleibt.

Dass Applikationen wie der Wahl-O-Mat von ohnehin bereits Mobilisierten stark genutzt werden, verringert jedoch den Mobilisierungseffekt in seiner Reichweite. Jeder Untersuchung der mobilisierenden Kapazität von Online-Anwendungen muss deswegen die Frage nach der Nutzerschaft und den damit verbundenen Kapazitäten eines Tools, politikferne Milieus zu erreichen, vorgeschaltet werden. Die Ergebnisse der Analyse sprechen deutlich für einen applikationsspezifischen Blickwinkel, der die Format- und Nutzungseigenschaften der jeweiligen InternetApplikation als Rahmenbedingungen für die Richtung und die Reichweite von Wirkungen berücksichtigt.

Somit sind die vorliegenden Ergebnisse nur bedingt auf „Online-Kommunikation “ generell beziehbar, die innerhalb des technisch bedingten Plattformformats eine Vielzahl von anwendungsspezifischen Unterformaten umfasst. Das Internet als technisches Basissystem und "Medium erster Ordnung“ beinhaltet zahlreiche „Medien zweiter Ordnung“, die durch „semiotische, institutionelle und organisatorische Heterogenität" geprägt sind (Beck 2010, S. 17) und deren Wirkungsweise folglich applikationsscharf ermittelt werden muss. Nichtsdestoweniger verweisen die Befunde auf Wirkungspotenziale eines „Internet-Mediums zweiter Ordnung “, die unter bestimmten Randbedingungen manifest werden können und politisch relevant sind. Diese Erkenntnisse gilt es für weitere Online-Anwendungen zu überprüfen.

Aber selbst die vorliegende Analyse eines ausgewählten Internet-Tools, des Wahl-O-Mat, hat noch Fragen offen gelassen, die einen verbleibenden Forschungsbedarf indizieren: So handelt es sich bei der verwendeten GLES-VorwahlBefragung um die Abfrage von Selbsteinschätzungen. Ob die Personen, die bekundeten, zur Wahl zu gehen, dies tatsächlich auch getan haben, konnte nicht nachgehalten werden. Auch ist die kausale Richtung des Zusammenhangs nicht durchweg eindeutig: Denkbar ist beispielsweise nicht nur, dass die Wahl-O-MatNutzung die Wahlbeteiligungsabsicht steigert, sondern dass eine sichere Wahlabsicht die Nutzung des Wahl-O-Mat wahrscheinlicher macht - weil man auf der Suche nach Orientierung ist, welche Partei man wählen soll. 
All diese offenen Fragen unterstützen die Forderung nach weiterer systematischer Erforschung dieser spezifischen Internet-Anwendungen und ihrer Implikationen mit alternativen Daten und Methoden. Wünschenswert wäre es, zur Erforschung des Wahl-O-Mat mit Daten zu arbeiten, die repräsentativ für die Gesamtbevölkerung sind und auf Zufallsstichproben basieren. Um nicht von Selbstauskünften abhängig zu sein, wäre überdies ein Panel-Design hilfreich, das die Effekte des Wahl-O-Mat auch nach den Wahlen nachhält - ähnlich der Untersuchung von Walgrave et al. aus dem Jahr 2006 (deren Sample allerdings nicht repräsentativ ist). Ergänzend könnten experimentelle Studien Erkenntnisse über den Wirkmechanismus von VAAs erbringen.

Da davon auszugehen ist, dass in zukünftigen Wahlkämpfen das Internet mit seinen Anwendungen eine stabile bis wachsende Rolle einnehmen wird, und vieles dafür spricht, dass die Issue-Orientierung der Wähler eine wichtige - wenn nicht gar wichtigere - Größe sein wird, werden Tools wie der Wahl-O-Mat an Relevanz für das Wahlverhalten gewinnen. Dies spricht für weitere Forschungsanstrengungen, um Wirkungen und Wirkungsweisen von Voting Advice Applications zu analysieren.

\section{Literatur}

Abold, Roland. 2006. The Audience is listening? Nutzung und Akzeptanz von Weblogs im Bundestagswahlkampf 2005. kommunikation@gesellschaft 7: Beitrag 1. http://www. soz.uni-frankfurt.de/K.G/B1_2006_Abold.pdf; zugegriffen: 08.11.2011.

Backhaus, Klaus, Bernd Erichson, Wulff Plinke und Rolf Weiber. 2003. Multivariate Analysemethoden. Eine anwendungsorientierte Einführung. Berlin: Springer Verlag.

Beck, Klaus. 2010. Soziologie der Online-Kommunikation. In Handbuch Online-Kommunikation, Hrsg. Wolfgang Schweiger und Klaus Beck, 15-35. Wiesbaden: VS Verlag für Sozialwissenschaften.

Best, Henning, und Christof Wolf. 2010. Logistische Regression. In Handbuch der sozialwissenschaftlichen Datenanalyse, Hrsg. Henning Best und Christof Wolf, 827-854. Wiesbaden: VS Verlag für Sozialwissenschaften.

Boogers, Marcel. 2006. Enquete bezoekers Stemwijzer. http://www.publiek-politiek.nl/content/ download/1976/10618/file/Enqu\%C3\%AAte\%20bezoe\%20kers\%20Stemwijzer.pdf; zugegriffen: 12.05.2011.

Brettschneider, Frank. 2005. Massenmedien und Wählerverhalten. In Handbuch Wablforschung, Hrsg. Jürgen W. Falter und Harald Schoen, 47-500. Wiesbaden: VS Verlag für Sozialwissenschaften.

Bryant, Jennings, und Mary Beth Oliver (Hrsg.). 2009. Media Effects. Advances in Theory and Research. New York: Routledge.

Caballero, Claudio. 2005. Nichtwahl. In Handbuch Wablforschung, Hrsg. Jürgen W. Falter und Harald Schoen, 329-365. Wiesbaden: VS Verlag für Sozialwissenschaften.

Cameron, A. Colin, und Frank A.G. Windmeijer. 1997. An R-squared Measure of Goodness of Fit for some Common Nonlinear Regression Models. Journal of Econometrics 77:329-342.

Campbell, Angus, Converse Philip E., Warren E. Miller und Donald E. Stokes. 1960. The American Voter. New York u.a.: Wiley. 
Cedroni, Lorella. 2010. Voting Advice Applications in Europe: A Comparison. In Voting Advice Applications in Europe. The State of the Art, Hrsg. Lorella Cedroni und Diego Garzia, 247-271. Napoli: Scriptaweb.

Cedroni, Lorella, und Diego Garzia (Hrsg.). 2010. Voting Advice Applications in Europe. The State of the Art. Napoli: Scriptaweb.

Chadwick, Andrew J. 2006. Internet Politics. States, Citizens and new Communication Technologies. New York: Oxford University Press.

Chadwick, Andrew J., und Philip N. Howard (Hrsg.). 2009. Routledge Handbook of Internet Politics. London: Routledge.

De Graaf, Jochum. 2010. The Irresistible Rise of Stemwijzer. In Voting Advice Applications in Europe. The State of the Art, Hrsg. Lorella Cedroni und Diego Garzia, 35-60. Napoli: Scriptaweb.

De Rosa, Roberto. 2010. cabina-elettorale.it (Provides advice to Italian voters since 2009). In Voting Advice Applications in Europe. The State of the Art, Hrsg. Lorella Cedroni und Diego Garzia, 187-212. Napoli: Scriptaweb.

Diaz-Bone, Rainer. 2006. Statistik für Soziologen. Konstanz: UVK.

Diaz-Bone, Rainer, und Harald Künemund. 2003. Einführung in die binäre logistische Regression. Mitteilungen aus dem Schwerpunktbereich Methodenlehre 56. Berlin: Institut für Soziologie der Freien Universität Berlin.

Downs, Anthony. 1957. An Economic Theory of Democracy. New York: Harper.

Drew, Dan, und David Weaver. 2006. Voter Learning in the 2004 Presidential Election: Did the Media Matter? Journalism and Mass Communication Quarterly 83:25-42.

Emmer, Martin, und Gerhard Vowe. 2004. Mobilisierung durch das Internet? Ergebnisse einer empirischen Längsschnittuntersuchung zum Einfluss des Internets auf die politische Kommunikation der Bürger. Politische Vierteljahresschrift 45:191-212.

Emmer, Martin. 2005. Politische Mobilisierung durch das Internet? Eine kommunikationswissenschaftliche Untersuchung zur Wirkung eines neuen Mediums. München: Verlag Reinhard Fischer.

Emmer, Martin, und Jens Wolling. 2010. Online-Kommunikation und politische Öffentlichkeit. In Handbuch Online-Kommunikation, Hrsg. Wolfgang Schweiger und Klaus Beck, 36-58. Wiesbaden: VS Verlag für Sozialwissenschaften.

Emmer, Martin, Angelika Füting und Gerhard Vowe. 2006. Wer kommuniziert wie über politische Themen. Eine empirisch basierte Typologie individueller politischer Kommunikation. Medien \& Kommunikationswissenschaft 54:216-236.

Faas, Thorsten. 2010. Das fast vergessene Phänomen. Hintergründe der Wahlbeteiligung bei der Bundestagswahl 2009. In Die Bundestagswahl 2009. Analysen der Wahl-, Parteien-, Kommunikations- und Regierungsforschung, Hrsg. Karl-Rudolf Korte, 69-86. Wiesbaden: VS Verlag für Sozialwissenschaften.

Farrell, David, und Rüdiger Schmitt-Beck (Hrsg.). 2008. Non-Party Actors in Electoral Politics. The Role of Interest Groups and Independent Citizens in Contemporary Election Campaigns. Baden-Baden: Nomos.

Fromm, Sabine. 2005. Binäre logistische Regressionsanalyse. Eine Einführung für Sozialwissenschaftler mit SPSS für Windows. Bamberger Beiträge zur empirischen Sozialforschung 11/2005, Hrsg. Gerhard Schulze und Leila Akremi. Bamberg: Universität Bamberg. 
Fuchs, Dieter, und Robert Rohrschneider. 2005. War es dieses Mal nicht die Ökonomie? Der Einfluss von Sachfragenorientierungen auf die Wählerentscheidung bei der Bundestagswahl 2002. In Wablen und Wähler. Analysen aus Anlass der Bundestagswabl 2002, Hrsg. Jürgen W. Falter, Oscar W. Gabriel, und Bernhard Weßels, 339-356. Wiesbaden: VS Verlag für Sozialwissenschaften.

Gabriel, Oscar W., Silke I. Keil und Isabell Thaidigsmann. 2009. Kandidatenorientierung und Wahlentscheid bei der Bundestagswahl 2005. In Wahlen und Wähler. Analysen aus Anlass der Bundestagswahl 2005, Hrsg. Oscar W. Gabriel, 267-303. Wiesbaden: VS Verlag für Sozialwissenschaften.

Gabriel, Oscar W., und Katja Neller. 2005. Kandidatenorientierungen und Wahlverhalten bei den Bundestagswahlen 1994-2002. In Wablen und Wähler. Analysen aus Anlass der Bundestagswahl 2002, Hrsg. Jürgen W. Falter, 213-243. Wiesbaden: VS Verlag für Sozialwissenschaften.

Garzia, Diego. 2010. The Effects of VAAs on Users' Voting Behaviour: An Overview. In Voting Advice Applications in Europe. The State of the Art, Hrsg. Lorella Cedroni und Diego Garzia, 13-47. Napoli: Scriptaweb.

Gibson, Rachel K., Wainer Lusoli und Stephen Ward. 2005. Online Participation in the UK: Testing a ,Contextualised' Model of Internet Effects. British Journal of Politics and International Relations 7:561-583.

Hirzalla, Fadi, Lisbet van Zoonen und Jan de Ridder. 2011. Internet Use and Political Participation: Reflections on the Mobilization/Normalization Controversy. The Information Society 27:1-15.

Johann, David. 2009. Eine Betrachtung der Wahlbeteiligung bei der Bundestagswahl 2005 auf Basis von Rational-Choice-Konzepten. In Wähler in Deutschland. Sozialer und politischer Wandel, Gender und Wablverhalten, Hrsg. Steffen Kühnel, Oskar Niedermayer und Bettina Westle, 424-449. Wiesbaden: VS Verlag für Sozialwissenschaften.

Johnson, Thomas J., und Barbara K. Kaye. 2003. A Boost or Bust for Democracy? How the Web Influenced Political Attitudes and Behaviors in the 1996 and 2000 Presidential Elections. The International Journal of Press/Politics 8:9-34.

Kellermann, Charlotte, und Hans Rattinger. 2007. Wirtschaftslage, Arbeitslosigkeit und zugeschriebene Regierungsverantwortung als Bestimmungsfaktoren des Wahlverhaltens bei den Bundestagswahlen 2002 und 2005. In Die Bundestagswabl 2005. Analysen des Wablkampfes und der Wahlergebnisse, Hrsg. Frank Brettschneider, Oskar Niedermayer und Bernhard Weßels, 369-393. Wiesbaden: VS Verlag für Sozialwissenschaften.

Kleinnijenhuis, Jan, und Anita M. J. van Hoof. 2008. The Influence of Internet Consultants. Beitrag präsentiert auf dem Annual Meeting of the International Communication Association, Montreal, 22.-26.05.2008.

Kunz, Volker, und Isabell S. Thaidigsmann. 2005. Die Relevanz von Themenorientierungen für das Wahlverhalten bei der Bundestagswahl 2002. In Wablen und Wähler. Analysen aus Anlass der Bundestagswahl 2002, Hrsg. Jürgen W Falter, Oscar W. Gabriel und Bernhard Weßels, 50-76. Wiesbaden: VS Verlag für Sozialwissenschaften.

Ladner, Andreas, Gabriela Felder, und Jan Fivaz. 2008. Are Voting Advice Applications (VAAs) more than Toys? First Findings on Impact and Accountability of VAAs. IDHEAP Working Paper 3/2008. Lausanne: Hochschulinstitut für öffentliche Verwaltung (IDHEAP). 
Ladner, Andreas, Gabriela Felder, Stefani Gerber und Jan Fivaz. 2010. Die politische Positionierung der europäischen Parteien im Vergleich. Eine Analyse der politischen Positionen der europäischen Parteien anlässlich der Wahlen des Europäischen Parlaments 2009 mit besonderer Berücksichtigung der Schweizer Parteien. Cahier de l'IDHEAP 252. Lausanne: Hochschulinstitut für öffentliche Verwaltung (IDHEAP).

Ladner, Andreas, und Joëlle Pianzola. 2010. Do Voting Advice Applications Have an Effect on Electoral Participation and Voter Turnout? Evidence from the 2007 Swiss Federal Elections. In Electronic Participation, Hrsg. Efthimios Tambouris, Anne Macintosh und Olivier Glassey, 211-224. Berlin, Heidelberg, New York: Springer.

Lewis-Beck, Michael S., William G. Jacoby, Helmut Norpoth und Herbert F. Weisberg. 2008. The American Voter Revisited. Ann Arbor: University of Michigan Press.

Lin, Carolyn A. 2009. Effects of the Internet. In Media Effects. Advances in Theory and Research, Hrsg. Jennings Bryant und Mary Beth Oliver, 567-591. New York: Routledge.

Marschall, Stefan. 2011. Wahlen, Wähler, Wahl-O-Mat. Aus Politik und Zeitgeschichte B 4: 40-46.

Marschall, Stefan, und Christian K. Schmidt. 2010. The Impact of Voting Indicators: The Case of the German Wahl-O-Mat. In Voting Advice Applications in Europe. The State of the Art, Hrsg. Lorella Cedroni und Diego Garzia, 65-104. Napoli: Scriptaweb.

Maurer, Marcus, und Olaf Jandura. 2009. Masse statt Klasse? Einige kritische Anmerkungen zu Repräsentativität und Validität von Online-Befragungen. In Sozialforschung im Internet. Methodologie und Praxis der Online-Befragung, Hrsg. Nikolaus Jackob, Harald Schoen und Thomas Zerback, 61-73. Wiesbaden: VS Verlag für Sozialwissenschaften.

Menard, Scott. 2002. Applied Logistic Regression Analysis. Thousand Oaks: Sage.

Mykkänen, Juri, und Tom Moring. 2006. Dealigned Politics Comes of Age? The Effects of Online Candidate Selectors on Finnish Voters. Beitrag präsentiert auf der Conference of Politics on the Internet: New Forms of Media for Political Action, Tampere, 24.25.11.2006.

Nuytemans, Michiel, Stefaan Walgrave, und Kris Descouwer. 2010. Do the Vote Test: The Belgian Voting Aid Application. In Voting Advice Applications in Europe. The State of the Art, Hrsg. Lorella Cedroni und Diego Garzia, 125-156. Napoli: Scriptaweb.

Pautz, Hartwig. 2010. The Internet, Political Participation and Election Turnout. A Case Study of Germany's www.abgeordnetenwatch.de. German Politics and Society 28: 156-175.

Ramonaite, Aine. 2010. Voting Advice Applications in Lithuania: Promoting Programmatic Competition or Breeding Populism? Policy \& Internet 2:117-147.

Rudi, Tatjana. 2011. Sachthemen und politische Streitfragen. In Zwischen Langeweile und Extremen: Die Bundestagswahl 2009, Hrsg. Hans Rattinger, Sigrid Roßteutscher, Rüdiger Schmitt-Beck und Bernhard Weßels, 179-190. Baden-Baden: Nomos.

Ruusuvirta, Outi, und Martin Rosema. 2009. Do Online Vote Selectors Influence Electoral Participation and the Direction of Vote? Beitrag präsentiert auf der European Consortium for Political Research (ECPR) General Conference, Potsdam, 10.-12.09.2009.

Schmitt-Beck, Rüdiger, und Christian Mackenrodt. 2009. Politikvermittlung durch Massenmedien bei der Bundestagswahl 2005: Nutzungsintensität und Einflüsse auf Einstel- 
lungen und Wahlverhalten. In Politik in der Mediendemokratie, Hrsg. Frank Marcinkowski, 415-446. Wiesbaden: VS Verlag für Sozialwissenschaften.

Schmitt-Beck, Rüdiger, Hans Rattinger, Sigrid Roßteutscher und Bernhard Weßels. 2010.

Die deutsche Wahlforschung und die German Longitudinal Election Study (GLES). In Gesellschaftliche Entwicklungen im Spiegel der empirischen Sozialforschung, Hrsg. Frank Faulbaum und Christof Wolf, 141-172. Wiesbaden: VS Verlag für Sozialwissenschaften.

Schnell, Rainer, Paul B. Hill und Elke Esser. 2008. Methoden der empirischen Sozialforschung. München: Oldenbourg.

Schoen, Harald, und Cornelia Weins. 2005. Der sozialpsychologische Ansatz zur Erklärung von Wahlverhalten. In Handbuch Wahlforschung, Hrsg. Jürgen W. Falter, 187-242. Wiesbaden: VS Verlag für Sozialwissenschaften.

Schoen, Harald. 2011. Merely a Referendum on Chancellor Merkel? Parties, Issues and Candidates in the 2009 German Federal Election. German Politics 20:92-106.

Schoen, Harald. 2010. Kampagnenrezeption und Beteiligung an der Europawahl 2009. Eine Analyse auf der Basis einer Onlineumfrage. In Die Massenmedien im Wablkampf. Das Superwahljahr 2009, Hrsg. Christina Holz-Bacha, 42-66. Wiesbaden: VS Verlag für Sozialwissenschaften.

Schoen, Harald. 2007. Campaigns, Candidate Evaluations, and Vote Choice: Evidence from German Federal Election Campaigns, 1980-2002. Electoral Studies 26:324-337.

Schulz, Winfried. 2010. Politische Kommunikation. Theoretische Ansätze und Ergebnisse empirischer Forschung. Wiesbaden: VS Verlag für Sozialwissenschaften.

Schweitzer, Eva J., und Steffen Albrecht (Hrsg.). 2011. Das Internet im Wablkampf. Analysen zur Bundestagswabl 2009. Wiesbaden: VS Verlag für Sozialwissenschaften.

Sharma, Subhash. 1996. Applied Multivariate Techniques. New York: John Wiley \& Sons.

Steinbrecher, Markus, und Hans Rattinger. 2011. Die Wahlbeteiligung. In Zwischen Langeweile und Extremen: Die Bundestagswahl 2009, Hrsg. Hans Rattinger, Sigrid Roßteutscher, Rüdiger Schmitt-Beck und Bernhard Weßels, 77-90. Baden-Baden: Nomos.

Taddicken, Monika. 2007. Methodeneffekte von Web-Befragungen - Freund oder Feind des Forschers? In Online-Forschung 2007. Grundlagen und Fallstudien, Hrsg. Martin Welker und Olaf Wenzel, 85-102. Köln: von Halem.

Tolbert, Caroline J., und Ramona S. McNeal. 2003. Unraveling the Effects of the Internet on Political Participation? Political Research Quarterly 56:175-185.

Trechsel, Alexander H., und Peter Mair. 2009. When Parties (also) Position Themselves: an Introduction to the EU Profiler. EUI RSCAS Working Paper 65. http://www.eui.eu/ Documents/RSCAS/Research/EUDO/RSCAS200965.pdf; zugegriffen: 11.05.2011.

Wagner, Aiko und Bernhard Weßels. 2012. Kanzlerkandidaten - Wie beeinflussen sie die Wahlentscheidung? In Wählen in Deutschland, Hrsg. Rüdiger Schmitt-Beck, 345-370. Baden-Baden: Nomos.

Walgrave, Stefaan, Peter van Aelst und Michiel Nuytemans. 2006. "Do the Vote Test". Electoral Effects of a Vote Advice Application at the 2004 Belgian Election. Beitrag präsentiert auf den ECPR Joint Workshop Sessions, Nikosia, 25.-30.04.2006.

Walgrave, Stefaan, Peter van Aelst und Michiel Nuytemans. 2008. "Do the Vote Test": The Electoral Effects of a Popular Vote Advice Application at the 2004 Belgian Elections. Acta Politica 43:50-70. 
Walgrave, Stefaan, Michiel Nuytemans und Koen Pepermans. 2009. Voting Aid Applications and the Effect of Statement Selection. West European Politics 32:1161-1180.

Wall, Matthew, Maria-Laura Sudulich, Rory Costello und Enricque Leon. 2009. Picking Your Party Online - An Investigation of Ireland's First Online Voting Advice Application. Information Polity 14:203-218.

Westle, Bettina, und Oskar Niedermayer. 2009. Orientierungen gegenüber der Demokratie. In Wähler in Deutschland. Sozialer und politischer Wandel, Gender und Wahlverhalten, Hrsg. Steffen Kühnel, Oskar Niedermayer und Bettina Westle, 11-29. Wiesbaden: VS Verlag für Sozialwissenschaften.

Zerback, Thomas, Harald Schoen, Nikolaus Jackob und Stefanie Schlereth. 2009. Zehn Jahre Sozialforschung im Internet - eine Analyse zur Nutzung von Online-Umfragen in den Sozialwissenschaften. In Sozialforschung im Internet. Methodologie und Praxis der Online-Befragung, Hrsg. Nikolaus Jackob, Harald Schoen und Thomas Zerback, 1531. Wiesbaden: VS Verlag für Sozialwissenschaften.

Zittel, Thomas, Dieter Fuchs (Hrsg.). 2007. Participatory Democracy and Political Participation. Can Participatory Engineering Bring Citizens Back In? New York: Routledge.

Zittel, Thomas, und Eilika Freund. 2011. Electoral Participation on the Internet - Mobilizing the Mobilized or Bringing Citizens Back In? Beitrag präsentiert auf der 6th ECPR General Conference, Reykjavik, 25.-27.08.2011.

\section{Autorenangaben:}

Prof. Dr. Stefan Marschall,

Heinrich-Heine-Universität Düsseldorf, Institut für Sozialwissenschaften, Universitätsstraße 1, 40225 Düsseldorf, stefan.marschall@uni-duesseldorf.de

[Korrespondenzautor]

Martin Schultze, M.A., Heinrich-Heine-Universität Düsseldorf, Institut für Sozialwissenschaften, Universitätsstraße 1, 40225 Düsseldorf, martin.schultze@uni-duesseldorf.de 